\title{
Does Young Maternal Age Have Any Adverse Effect on Maternal and Fetal Health? A Retrospective Case Control Study
}

\section{Erdem SAHIN1, Yusuf MADENDAG ${ }^{2}$, Mefkure ERASLAN SAHIN¹, Ilknur COL MADENDAG², Gokhan ACMAZ², Iptisam Ipek MUDERRIS ${ }^{3}$}

Kayseri, Turkey

\begin{abstract}
OBJECTIVE: To reveal whether adolescent mothers are at higher risk of poor fetal and maternal outcomes compared with non-adolescent mothers.

STUDY DESIGN: A total of 426 nulliparous women having a single pregnancy and gave birth with a birth-weight $>500 \mathrm{~g}$ or $>24$ weeks' gestation were included. Participants were divided into three groups. 1. Group consisted of adolescents between ages of 16-17 $(n=56), 2$. Group 18-19 ages $(n=185)$ and 3 . Group 20-24 ages $(n=185)$. Adverse maternal and fetal outcomes compared among groups.

RESULTS: The mean maternal age was 16.6( \pm 0.40$), 18.1( \pm 0.55), 22.6( \pm 0.60)$ in groups, respectively. Antenatal visits number, smoking status, vaginal delivery, emergency cesarean delivery rates (fetal distress or cephalopelvic disproportion), perineal infection, postpartum hemorrhage, transfusion requirement, maternal death were not statistically significant among groups $(p=0.871, p=0.935, p=0.450$, $p=0.961, p=0.737, p=0.112, p=0.996, p=0.859$ and $p=1$, respectively). Induction of labour, delivery $<37$ weeks, low birth weight $(<2500 \mathrm{~g})$, admission to $\mathrm{NICU}$, meconium-stained amniotic fluid, severe neonatal conditions, respiratory distress syndrome, hypoxic ischemic encephalopathy, neonatal death rates were not statistically significant among groups $(p=0.187, p=0.560, p=0.408, p=0.347, p=0.901, p=0.605$, $p=1, p=1$ and $p=1$, respectively).

CONCLUSIONS: The data from the present study indicates that when the adolescent period is grouped not as a whole but within itself, especially low gynecological age is associated with increased adverse fetal outcomes, and it does not carry any risk for the mother. Pregnancy in adolescents aged 18-19 years does not constitute a risk for both mother and child. If it is thought that an adolescent pregnant woman can not give birth vaginally, the cesarean ratio will increase unnecessarily. Hence maternal age should not be a criterion singly for cesarean delivery.
\end{abstract}

Keywords: Adolescent pregnancy, Teen mother, Adverse maternal outcomes, Adverse fetal outcomes

Gynecol Obstet Reprod Med 2018;24(2):60-64

1 Department of Obstetrics and Gynecology University of Health Sciences Sarkisla Government Hospital, Sivas

2 Department of Obstetrics and Gynecology Kayseri Education and Research Hospital, Kayseri

${ }^{3}$ Department of Obstetrics and Gynecology Erciyes University Medicine Faculty, Kayseri

Address of Correspondence: Yusuf Madendag

University of Health Sciences Kayseri

Education and Research Hospital,

38039 Kayseri, Turkey

yusufmadendag@gmail.com

Submitted for Publication: $\quad 30.09 .2017$

Accepted for Publication: $\quad$ 19.11.2017

\begin{tabular}{|c|c|}
\hline \multicolumn{2}{|c|}{ Access this article online } \\
\hline \multirow{2}{*}{ 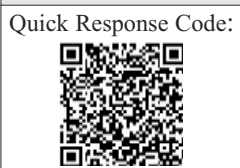 } & $\begin{array}{l}\text { Website: www.gorm.com.tr } \\
\text { e mail: info@gorm.com.tr }\end{array}$ \\
\hline & DOI:10.201613/GORM.217.740 \\
\hline
\end{tabular}

How to cite this article: Sahin E. Madendag Y. Eraslan Sahin M. Col Madendag I. Acmaz G. Muderris I. Does Young Maternal Age Have Any Adverse Effect on Maternal and Fetal Health? A Retrospective Case Control Study. Gynecol Obstet Reprod Med 2018:24(2):60-64

\section{Introduction}

Adolescent pregnancy is described as a pregnancy in aged 10-19 years. Births to adolescents mothers represent $10 \%$ of births worldwide, but $23 \%$ of maternal morbidity and mortality (1). Delivery in adolescence is not only related to adverse perinatal outcomes but also affects the potential well-being of both the infant and the mother.

Prior reports revealed that adolescent pregnancy was associated with higher incidence of low birthweight, preterm delivery, perinatal and maternal death (2-4). Nevertheless, there were inconsistent results from earlier reports as to whether the adverse pregnancy outcomes of adolescent mothers were triggered by their lack of biological maturity or poor socio-environmental factors $(2,5)$. It is difficult to compare the results of adolescent pregnancy due to the heterogeneity amongst survey settings, the number of participants being low, particularly 
for younger ( $\leq 15$ years) adolescent mothers, the quality of the hospital and pregnant women's social class.

In the present study, we aimed to investigate whether adolescent mothers are at higher risk of adverse fetal and maternal outcomes compared with non-adolescent mothers.

\section{Material and Method}

This study was conducted, retrospectively, in Obstetrics and Gynecology Department at the Health Sciences University Kayseri Education and Research Hospital in Kayseri, Turkey. It was approved by the Ethics Committee of Erciyes University (decision number: 2017/396) and was carried out by the Declaration of Helsinki.

A total of 426 nulliparous pregnant women aged between 16 and 24 years, who had a single pregnancy and gave birth to an infant of at least 24 weeks' gestation or with a birth-weight of at least $500 \mathrm{~g}$ were included. Participants were divided into three groups. The first group consisted of adolescents between the ages of 16-17, the second group consisted of adolescents between the ages of 18-19 and the third group consisted of non-adolescents between the ages 20-24. In our analysis, we selected mothers aged 20- 24 years as the reference group, consistent with previous studies $(6,7)$.

It has also been suggested that girls who conceive shortly after menarche $(<2$ years after menarche, defined as low gynecological age) are at an increased risk of adverse outcomes (8). As menarche tends to start later in girls with poor nutritional and environmental conditions, it was assumed that many adolescent mothers aged 16-17 years would have a low gynecological age and therefore it may not be appropriate to categories them with older adolescents aged 18-19 years. Hence the maternal age was categorized into three groups: 16$17,18-19$ and 20-24 years in this study.
We excluded external factors, such as violence, accident, poisoning or self-harm which affect maternal and fetal wellbeing. Also, patients were not included in the study in cases of multiple pregnancies, preeclampsia, gestational hypertension, chronic hypertension, chronic kidney, liver and heart disease, gestational diabetes, type 1 and type 2 diabetes, alcohol and drug use.

Maternal characteristic, mode of delivery, number of antenatal visits and smoking status were recorded. Adverse perinatal outcomes were defined as the presence any of the following; postpartum hemorrhage, perineal infection, transfusion requirement and maternal death. Adverse perinatal outcomes were defined as the presence any of the following; prematurity, Apgar 5 min $<7$, emergency cesarean delivery for fetal distress, respiratory distress syndrome (RDS), meconium-stained amniotic fluid, admission to the neonatal intensive care unit (NICU) and length of stay on the NICU, hypoxic-ischemic encephalopathy (HIE) and neonatal death.

Statistic analysis: The Shapiro-Wilk test was used to test the normality assumption of the data. Levene test was used to the variance homogeneity assumption. Values are expressed usually as $n(\%)$, but a mean \pm standard deviation was used only for age parameter. Parametric comparisons were made using a t-test or z-test, and nonparametric comparisons were made using the Mann-Whitney U test. All comparisons were made with the PASW Statistics 18 program and p-value of $<0.05$ was considered statistically significant.

\section{Results}

Overall 426 nulliparous pregnant women were enrolled in the study. 56 of them were 16-17 years old, 185 of them were 18-19 years old, and 185 of them were 20-24 years old. The comparison of maternal characteristic and adverse maternal outcomes is shown in table 1 .

Table 1: Comparison of adverse maternal outcomes among groups.

\begin{tabular}{|c|c|c|c|c|}
\hline & \multicolumn{3}{|c|}{ Maternal Age } & \multirow[b]{2}{*}{$\begin{array}{c}p \\
\text { Value }\end{array}$} \\
\hline & $\begin{array}{c}16-17 \text { years } \\
(n: 56)\end{array}$ & $\begin{array}{c}18-19 \text { years } \\
(n: 185)\end{array}$ & $\begin{array}{c}\text { 20-24 years } \\
(n: 185)\end{array}$ & \\
\hline Maternal age (year) & $16.6( \pm 0.40)$ & $18.1( \pm 0.55)$ & $22.6( \pm 0.60)$ & $<0.001$ \\
\hline Antenatal visits number $\geq 10(\%)$ & $51(\% 91.07)$ & $170(\% 91.89)$ & $172(\% 92.97)$ & 0.871 \\
\hline Smoking status $(\%)$ & $4(\% 7.14)$ & $15(\% 8.11)$ & $16(\% 8.65)$ & 0.935 \\
\hline Vaginal delivery $(\%)$ & $51(\% 91.07)$ & $162(\% 87.57)$ & $157(\% 84.86)$ & 0.450 \\
\hline Emergency cesarean delivery for Fetal distress (\%) & $2(\% 3.57)$ & $6(\% 3.24)$ & $7(\% 3.78)$ & 0.961 \\
\hline Emergency cesarean delivery for Cephalopelvic disproportion (\%) & $0(\% 0)$ & $2(\% 1.08)$ & $2(\% 1.08)$ & 0.737 \\
\hline Perineal infection (\%) & $3(\% 5.36)$ & $3(\% 1.62)$ & $2(\% 1.08)$ & 0.112 \\
\hline Postpartum hemorrhage (\%) & $1(\% 1.79)$ & $3(\% 1.62)$ & $3(\% 1.62)$ & 0.996 \\
\hline Transfusion requirement (\%) & $0(\% 0)$ & $1(\% 0.54)$ & $1(\% 0.54)$ & 0.859 \\
\hline Maternal death $(\%)$ & $0(\% 0)$ & $0(\% 0)$ & $0(\% 0)$ & 1 \\
\hline
\end{tabular}

Different superscript numbers indicate statistically significant differences.

Values are expressed as mean \pm standard deviation or $n(\%)$. 
The mean maternal age was $16.6( \pm 0.40)$ in group 1, $18.1( \pm 0.55)$ in group 2 and $22.6( \pm 0.60)$ in group 3. Antenatal visits number, smoking status, vaginal delivery, emergency cesarean delivery rates (fetal distress or cephalopelvic disproportion), perineal infection, postpartum hemorrhage, transfusion requirement, maternal death ratio were not statistically significant among groups. $(\mathrm{p}=0.871, \mathrm{p}=0.935, \mathrm{p}=0.450$, $\mathrm{p}=0.961, \mathrm{p}=0.737, \mathrm{p}=0.112, \mathrm{p}=0.996, \mathrm{p}=0.859$ and $\mathrm{p}=1$, respectively).

The comparison of adverse fetal outcomes was shown in table 2. Induction of labor, delivery $<37$ weeks, low birth weight $(<2500 \mathrm{~g})$, admission to NICU, meconium-stained amniotic fluid, severe neonatal conditions, RDS, HIE, neonatal death rates were not statistically significant among groups. $(\mathrm{p}=0.187, \mathrm{p}=0.560, \mathrm{p}=0.408, \mathrm{p}=0.347, \mathrm{p}=0.901, \mathrm{p}=0.605$, $\mathrm{p}=1, \mathrm{p}=1$ and $\mathrm{p}=1$, respectively).

\section{Discussion}

Adolescence is a distinct physical and developmental stage in a women's life. Metabolic hemodynamic and psychological burden of pregnancy imposed on the adolescent are known to make the pregnancy risky for the mother whose reproductive physiology has not been matured yet.

In this study, we aimed to investigate the relationship between younger maternal age with unfavorable maternal and fetal outcomes. According to data from this study, it is seen that when the adolescent period is grouped not as a whole, but within itself, smaller gynecological age group is associated with adverse fetal outcomes and does not convey a risk for mothers. Adolescent pregnancies at 18 and 19 years of age do not constitute a risk both for the mother and child.

In the present study, we found that adolescent mothers were tended to have a vaginal delivery and had a similar risk of cesarean section, which was in harmony with prior reports
$(3,9,10)$. de Vienne et al. revealed that younger maternal age was related with increased risk of fetal death and decreased risks of preeclampsia, and post-partum hemorrhage in their study which included 8514 primiparous women aged less than 31 (3). Zeteroglu et al. reported that the cesarean deliveries are decreased in adolescent pregnancies and biological immaturity is not a significant problem in their study performed with 40,391 pregnant women; however, they compared adolescent mothers to non-adolescents between the ages of 18-35 and $\geq 35$ years (10). The age range of the control group is very wide, hence the comparison of groups with different physiological characteristics not appropriate and make it difficult to assess the age effect. Data from the present study has been shown that obstructed labor and caesarean section indicated for cephalo-pelvic disproportion because of the immaturity of the pelvic bone are more frequent in adolescents; however, studies performed in recent years where other factors have been ruled out, have demonstrated that cephalo-pelvic disproportion is less frequently seen in adolescents when compared with adults and accordingly birth traumas are less frequently seen and surgical delivery and cesarean section are required at a lower rate $(11,12)$.

In the current study, we found that there is no significant difference with postpartum hemorrhage and transfusion requirement among groups. This situation can be explained by the presence of higher amount of functional myometrial tissue in adolescent pregnant women, lower requirement for induction of labor and average birth weight (11). Also, perineal infection rates were comparable higher in adolescent pregnant women. According to the data of the present study inability of adolescent pregnant women to achieve adequate hygienic conditions or inadequacy of pelvic blood circulation during the adolescent period can be explained by the presence of the predisposing factors for infection (3).

In the current study, it was found that adolescent preg-

Table 2: Comparison of adverse fetal outcomes among groups.

\begin{tabular}{|c|c|c|c|c|}
\hline & \multicolumn{3}{|c|}{ Maternal Age } & \multirow[b]{2}{*}{$\begin{array}{c}p \\
\text { Value }\end{array}$} \\
\hline & $\begin{array}{c}\text { 16-17 years } \\
(n: 56)\end{array}$ & $\begin{array}{c}\text { 18-19 years } \\
(n: 185)\end{array}$ & $\begin{array}{c}\text { 20-24 years } \\
\text { (n: 185) }\end{array}$ & \\
\hline Induction of labor & $6(\% 10.71)$ & $22(\% 11.89)$ & $33(\% 17.84)$ & 0.187 \\
\hline Delivery<37 weeks & $5(\% 8.93)$ & $16(\% 8.65)$ & $11(\% 5.95)$ & 0.560 \\
\hline Low birth weight $(<2500 \mathrm{~g})$ & $8(\% 14.29)$ & $24(\% 12.97)$ & $17(\% 9.19)$ & 0.408 \\
\hline Admission to NICU & $4(\% 7.14)$ & $11(\% 5.95)$ & $6(\% 3.24)$ & 0.347 \\
\hline Meconium-stained amniotic fluid & $1(\% 1.79)$ & $2(\% 1.08)$ & $2(\% 1.08)$ & 0.901 \\
\hline Neonatal severe conditions & $2(\% 3.57)$ & $3(\% 1.62)$ & $3(\% 1.62)$ & 0.605 \\
\hline RDS & $0(\% 0)$ & $0(\% 0)$ & $0(\% 0)$ & 1 \\
\hline HIE & $0(\% 0)$ & $0(\% 0)$ & $0(\% 0)$ & 1 \\
\hline Neonatal death & $0(\% 0)$ & $0(\% 0)$ & $0(\% 0)$ & 1 \\
\hline
\end{tabular}

RDS: Respiratory distress syndrome, NICU: Neonatal intensive care unit, HIE: Hypoxicischemic encephalopathy, NA: Not available. Neonatal severe conditions refer to a live born baby with one of the following conditions: birthweight $<1500$ g. $<32$ gestational weeks at birth or a 5-minute Apgar score of $<7$. Values are expressed as $n(\%)$. 
nancy was not a risk for adverse perinatal outcomes. Consistent with previous studies although not statistically significant we found a higher ratio of low birth weight, preterm delivery and NICU admission $(4,6,13,14)$.

Mukhopadhyay et al. reported that the teenage mothers had more adverse perinatal complications, such as preterm births, stillbirths, neonatal deaths and delivered low birthweight babies when compared with those of the adult primigravid mothers. They compared with teenage mothers aged 13-19 years were regarded as the cases while adult mothers aged 20-29 years formed the comparison group. It was assumed that many adolescent mothers aged 16-17 years would have a low gynecological age and the physiological condition is further immaturized under the age of 16 years. Therefore it may not be appropriate to categories them with older adolescents aged 18-19 years. Increased adverse outcomes can be explained by the inclusion of adolescents under the age of 16 (4). Conde-Agudelo et al. evaluated 854.377 Latin American women who were younger than 25 years, and they reported adolescents had higher risks for postpartum hemorrhage, puerperal endometritis, operative vaginal delivery, episiotomy, low birth weight, preterm delivery and small-for-gestational-age infants and adolescent mothers had lower risks for cesarean delivery, third-trimester bleeding, and gestational diabetes. When one considers that the factors which might effect perinatal outcomes as SGA and gestational diabetes were not excluded in their studies, it will not be appropriate to state that only age is influential on outcomes (6). In another study, Omar et al. demonstrated that adolescent pregnancy was associated with low Apgar scores and perinatal complications. However, they compared adolescent mother aged 10-19 years to 20-35 years' non-adolescent mothers (13). In a study which included 51,890 women aged 13-24 years, arranged into three groups: $<$ or $=17,18-19$ and 20-24. Haldre et al. reported that risk of low birth weight and preterm birth was higher among teenagers. Increased risks of neonatal and post neonatal death among younger teenagers of an age of 17 years and less seem to be a result of prematurity (14). Previous studies have also suggested that these risks among adolescent mothers are associated with biological immaturity independent of poor socialeconomic status, smoking and inadequate antenatal care $(14$, 15). Gynecologic immaturity (i.e., short cervix $[\leq 25 \mathrm{~mm}]$, small uterine volume) and susceptibility to subclinical infections increase the risk of preterm delivery among adolescent mothers (16).

This study has some strengths; 1) although the adolescent period is defined as the time interval between 10 and 19 years of age, this age bracket carries different physiologic characteristics within itself. In our study, we did not classify the adolescent period as a whole, but based on their physiologic characteristics and compared adolescent age group with an age bracket of 20-24 years. 2) Environmental conditions are known to effect perinatal outcomes. In this study, we excluded external factors which will affect the well-being of fetus as violence, accident, poisoning of self-harm and evaluated only the effects of age on fetal and maternal outcomes. 3) We included only nulliparous adolescents in the study and control groups to rule out potential effects of the number of pregnancies on maternal and fetal outcomes.

\section{Conclusion}

In conclusion, the data from the present study indicates that; when the adolescent period is not grouped as a whole, but within itself, especially low gynecological age is associated with increased adverse fetal outcomes, and it does not convey risk for the mother. Adolescent pregnancies in women aged 18-19 years; do not constitute a risk for both mother and fetus. If it is thought that an adolescent pregnant woman can not give birth vaginally, the cesarean ratio will increase unnecessarily. Hence maternal age should not be a criterion singly for cesarean delivery. Further prospective studies with a greater number of patients are required in this regard.

\section{: Acknowledgments: None \\ Source of funding: None \\ Grant support \& financial disclosures: None \\ Conflicts of interest: The authors report no conflicts of inter- est.}

\section{References}

1. UNFPA. Annual report 2007. U.S.A: UNFPA; 2008. Available from: http://www.unfpa.org/sites/default/files/pubpdf/ar07_eng.pdf

2. Chen CW, Tsai CY, Sung FC, Lee YY, Lu TH, Li CY, et al. Adverse birth outcomes among pregnancies of teen mothers: age-specific analysis of national data in Taiwan. Child Care Health Dev 2010; 36(2):232-40.

3. de Vienne CM, Creveuil C, Dreyfus M. Does young maternal age increase the risk of adverse obstetric, fetal and neonatal outcomes: a cohort study. Eur J Obstet Gynecol Reprod Biol 2009;147(2):151-6.

4. Mukhopadhyay P, Chaudhuri RN, Paul B. Hospital-based perinatal outcomes and complications in teenage pregnancy in India. J Health Popul Nutr 2010;28(5):494-500.

5. Loto O, Ezechi O, Kalu B, Loto AB, Ezechi LO, Ogunniyi S. Poor obstetric performance of teenagers: is it age-or quality of care-related? J Obstet Gynecol 2004;24(4):395-8.

6. Conde-Agudelo A, Belizan JM, Lammers C. Maternalperinatal morbidity and mortality associated with adolescent pregnancy in Latin America: cross-sectional study. Am J Obstet Gynecol 2005;192(2):342-9.

7. Sharma V, Katz J, Mullany LC, Khatry SK, LeClerq SC, Shrestha SR, et al. Young maternal age and the risk of neonatal mortality in rural Nepal. Arch Pediatr Adolesc Med 2008;162(9):828-35. 
8. Gibbs CM, Wendt A, Peters S, Hogue CJ. The impact of early age at first childbirth on maternal and infant health. Paediatr Perinat Epidemiol 2012;26(s1):259-84.

9. Zeteroglu S, Sahin I, Gol K. Cesarean delivery rates in adolescent pregnancy. Eur J Contracept Reprod Health Care 2005;10(2):119-22.

10. Topçu HO, Güzel Aİ, Kokanalı K, Cavkaytar S, Uygur D, Erkaya S. Maternal Serum Nitric Oxide Levels in Adolescent Pregnancies. Gynecol Obstet Reprod Med 2016;20(2):92-95.

11. Thaithae S, Thato R. Obstetric and perinatal outcomes of teenage pregnancies in Thailand. J Pediatr Adolesc Gynecol 2011;24(6):342-6.

12. Keskinoglu P, Bilgic N, Picakciefe M, Giray H, Karakus $\mathrm{N}$, Gunay T. Perinatal outcomes and risk factors of Turkish adolescent mothers. J Pediatr Adolesc Gynecol
2007;20(1):19-24.

13. Omar K, Hasim S, Muhammad NA, Jaffar A, Hashim SM, Siraj HH. Adolescent pregnancy outcomes and risk factors in Malaysia. Int J Gynaecol Obstet 2010;111(3):220-3.

14. Haldre K, Rahu K, Karro H, Rahu M. Is a poor pregnancy outcome related to young maternal age? A study of teenagers in Estonia during the period of major socio-economic changes (from 1992 to 2002). Eur J Obstet Gynecol Reprod Biol 2007;131(1):45-51.

15. Thato S, Rachukul S, Sopajaree C. Obstetrics and perinatal outcomes of Thai pregnant adolescents: a retrospective study. Int J Nurs Stud 2007;44(7):1158-64.

16. Stevens-Simon C, Beach RK, McGregor JA. Does incomplete growth and development predispose teenagers to preterm delivery? A template for research. J Perinatol 2002;22(4):315-23 